medgen $2011 \cdot 23: 352-353$

DOI 10.1007/s11825-011-0286-8

Online publiziert: 1. Oktober 2011

(c) Springer-Verlag 2011

\author{
C. Fischer ${ }^{1} \cdot$ T. Grimm ${ }^{2}$ \\ ${ }^{1}$ Institut für Humangenetik, Universität Heidelberg \\ ${ }^{2}$ Abteilung für medizinische Genetik im Institut für Humangenetik, \\ Universität Würzburg
}

\title{
Risikoberechnungen in der Humangenetik
}

Die meisten Ereignisse in unserem Leben sind zufallsbehaftet. Wir wissen nicht, ob wir diese oder jene Krankheit irgendwann einmal bekommen werden und wie ihr Verlauf dann sein wird. Trotzdem lassen wir uns impfen oder nehmen ein Medikament, wenn es zum Beispiel mit 90\%iger Wahrscheinlichkeit gegen unsere Krankheit hilft. Die Wahrscheinlichkeit wird als Maß für unsere Sicherheit über das Eintreten eines Ereignisses im Licht der bisher angesammelten Erfahrungen - also im bayesianischen Sinn - interpretiert. Weder reiche noch arme Ratsuchende würden heutzutage das Orakel von Delphi befragen oder Würfel für ihre Entscheidung benutzen, wenn eine sog. objektive Wahrscheinlichkeit verlässlich bestimmt werden kann. Dies ist die Grundlage der evidenzbasierten Medizin und sie betrifft bekanntlich die Therapieentscheidung ebenso wie die Diagnostik und die Krankheitsprognose.

\section{( Die meisten Ereignisse in unserem Leben sind zufallsbehaftet}

Humangenetische Beratungen können jedoch vergleichbar mit dem Orakel von Delphi werden, wenn falsche genetische Modelle benutzt werden, falsche Berechnungen durchgeführt werden oder in komplexen Situationen unser noch eingeschränktes Wissen falsch interpretiert wird (s. auch den Beitrag von Cremer u. Propping: „Ziele und Grenzen der Quantifizierung genetischer Risiken“). Können dagegen die in der Realität beobachteten Gesetzmäßigkeiten hinreichend gut durch ein statistisches Modell beschrieben werden, kann man Wahrscheinlichkeiten für Ereignisse beziffern.

Die Grundlagen der Wahrscheinlichkeitsberechnungen werden in dem Beitrag von Fischer und Grimm: „Das Rechnen mit Wahrscheinlichkeiten in der $\mathrm{Hu}$ mangenetik“ kurz dargestellt. In der $\mathrm{Hu}$ mangenetik sind wir in der günstigen Lage, eine sehr mächtige Theorie zur Verfügung zu haben, nämlich die Mendelschen Regeln, mehr noch die haplotypbasierte Vererbung von einer Generation in die nächste. Bei monogenen Krankheiten und bei komplexen Krankheiten mit Hauptgenen sind genetisch-statistische Modelle formuliert worden und werden für Risikoberechnungen in der genetischen Beratung eingesetzt. Davon handeln 3 Beiträge in dieser Ausgabe (Fischer und Grimm: „Risikoberechnungen beim autosomaldominantem Erbgang“; Grimm et al.: „Risikoberechnungen beim autosomal-rezessiven Erbgang“; Grimm und Fischer: „Risikoberechnungen beim X-chromosomal-rezessiven Erbgang"). Eine Grundlage dieser Beiträge sind die von Fischer und Grimm erstellten Unterlagen des seit vielen Jahren regelmäßig stattfindenden Kurses „Risikoberechnungen in Familien" der Akademie Humangenetik. Genetische Berater wenden diese Techniken vorwiegend für Ratsuchende an, in deren Familien bereits ein hohes A-priori-Risiko

Die wissenschaftliche Koordination des vorliegenden Themenschwerpunkts erfolgte unter Leitung von Dr. sc. hum. Christine Fischer, Heidelberg, und Prof. Dr. med. Tiemo Grimm, Würzburg. 
vorliegt, mit dem Ergebnis, entweder eine noch höhere Wahrscheinlichkeit zu erhalten oder eine deutlich geringere.

Im Gegensatz dazu findet man für komplexe genetische Erkrankungen in genomweiten Assoziationsstudien viele genetische Varianten, die einzeln betrachtet oft nur einen geringen Anteil zum Erkrankungsrisiko beitragen. Auch zusammengefasst sind sie in ihrer Vorhersagekraft auf Populationsebene für viele Volkskrankheiten enttäuschend, können aber in Hochrisikogruppen möglicherweise hilfreich sein. Diesem Thema ist der Beitrag von Heid et al. gewidmet: „Wie groß sind die kleinen genetischen Risiken?" Ob eine genetische Disposition zu einem höheren Erkrankungsrisiko führt, hängt von den Auswirkungen weiterer ungünstiger Umwelteinflüsse und von epigenetischen Mechanismen ab, die bisher nur in ihren Ansätzen bekannt sind (s. Cremer u. Propping: „Ziele und Grenzen der Quantifizierung genetischer Risiken“). Der Beitrag von Krawczak: „Rauchen und Lungenkrebs - Wie falsch lag Fisher wirklich?" beschäftigt sich mit dem Problem, dass statistische Assoziation allein uns nichts über Kausalität vermitteln kann, und mit der möglichen Verzerrung durch unbekannte Confounder.

Für monogene Krankheiten gibt es etliche verkomplizierende Faktoren wie etwa genetische und allelische Heterogenität sowie variable Expressivität, auf die wir hier nicht ausführlicher eingehen können. Insbesondere bei komplexen genetischen Krankheiten müssen Risikovorhersagen vor einem Alltagseinsatz in Studien prospektiv auf ihre klinische „Wirksamkeit“ überprüft werden. Darüber entscheiden die Vorhersageeigenschaften und die Präventions- und Therapieoptionen sowie die klinischen Konsequenzen falscher Entscheidungen.

Wir hoffen, dass dieses Themenheft „Risikoberechnungen in der Humangenetik“ in der täglichen Praxis der Humangenetik hilfreich sein wird.

\section{Korrespondenzadresse}

\section{Dr. sc. hum. C. Fischer}

Institut für Humangenetik

Universität Heidelberg

Im Neuenheimer Feld 366

69120 Heidelberg

c.fischer@uni-hd.de

\section{Prof. Dr. T. Grimm}

Abteilung für medizinische Genetik im Institut für Humangenetik Universität Würzburg, Biozentrum Am Hubland, 97074 Würzburg tgrimm@biozentrum.uni-wuerzburg.de 